\title{
PROGRAMA DE FORMACIÓN DE POSGRADO SOBRE GESTIÓN DE LA INTERNACIONALIZACIÓN DE LA EDUCACIÓN SUPERIOR - GIES
}

\author{
Universidad Nacional de Cuyo (UN- \\ CUYO, Mendoza, Argentina) Facul- \\ tad de Filosofía y Letras
}

DOI: $10.25087 /$ resur8a4

\section{Presentación sintética de la propuesta}

El Programa de Gestión de Internacionalización de la Educación Superior surge como iniciativa por parte de la Facultad de Filosofía y Letras de la Universidad Nacional de Cuyo para establecer redes con universidades latinoamericanas y del mundo en la formación de gestores de la internacionalización.

En un contexto mundial en el que los procesos de nacionalización, regionalización e internacionalización de la educación en sus diferentes niveles son crecientes, es necesaria la optimización de los mecanismos de movilidad de los actores educativos. En este marco, las universidades, con sus funciones de docencia, investigación y extensión, son protagonistas en la búsqueda de los medios más efectivos para la movilidad de docentes, estudiantes y gestores.

Por este motivo, la formación, la actualización y el intercambio de experiencias en gestión de la internacionalización de la educación superior, resulta de utilidad para aquellas instituciones interesadas en la construcción de redes de cooperación educativa.

En este sentido, el presente documento es el resultado, por un lado, de una evaluación diagnóstica elaborada durante el año 2017, en el que se realizó una prueba piloto de la propuesta formativa en gestión de la internacionalización de la educación superior, en la que participaron gestores de 5 países latinoamericanos. Por otro lado, el documento recoge los resultados de la Jornada de intercambio y discusión en torno al Programa GIES, que tuvo lugar el 29 de julio de 2019 en la Facultad de Filosofía y Letras de la Universidad Nacional de Cuyo. 
Programa de formación de posgrado sobre gestión de la internacionalización de la educación superior GIES

\section{Objetivos}

- Formar y/o perfeccionar a actores y/o investigadores que se dediquen a la gestión de la nacionalización, regionalización y/o internacionalización de la Educación Superior, teniendo en cuenta todas sus dimensiones y complejidad.

- Generar un espacio interinstitucional de cooperación en integración, en América Latina y el Caribe, para promover buenas prácticas en gestión de la Internacionalización de la Educación Superior, acorde a las necesidades y demandas regionales e internacionales.

- Proponer la investigación en general, y la investigación desde la educación comparada, en particular, sobre temas relacionados con los procesos de integración educativa desde la perspectiva internacional; así como difundir los conocimientos que de ellas surjan.

- Definir políticas del conocimiento en materia de gestión de la Internacionalización de la Educación Superior, para responder a las necesidades y demandas regionales e internacionales.

- Fortalecer los sistemas de información para la transparencia con el fin de elaborar bases de datos y sistemas de apoyos, sobre recursos y potencialidades locales, regionales e internacionales.

\section{Responsables del Programa}

Dirección: Dra. Marisa Carina Fazio (FFyL- UNCuyo)

Co- Dirección: Dra. Natalia Coppola

Coordinación Ejecutiva:

Mg. Virginia Paez (FFyL, UNCuyo)

Esp. Miriam Fernández (FFyL-UNCuyo)

\section{Comité Académico:}

Presidente: Dr. Adolfo Omar Cueto (Facultad de Filosofía y Letras, UNCuyo)

Dirección: Dra. Marisa Carina Fazio (Facultad de Filosofía y Letras, UNCuyo)

Dr. Augusto Pérez Lindo

Dr. Paulo Falcón 

Dr. Ángel Tuninetti
Dr. Jeferson Diel
Dra. Raquel Morales
Dr. Luis González
Dr. Enrique Martínez Larrechea

\section{Comité Ejecutivo:}

- Dr. Jeferson Odiel Dier (Universidade do Estado do Mato Grosso) Brasil.

- Dra. María Raquel Morales Alarcón (Universidad Bernardo O’Higgins) Chile.

- Dr. Enrique Martínez Larrechea (Instituto Universitario Sudamericano) Uruguay

\section{Destinatarios:}

Actores interesados en la gestión de la Internacionalización de la Educación Superior de distinta instituciones públicas, privadas y Sociedad Civil.

\section{Modalidad}

Los Módulos se dictarán utilizando como soporte la plataforma de la FFyL - UNCuyo, con tutores virtuales disciplinares y no disciplinares. A su vez, se deberá acreditar la asistencia en las actividades previstas en las Instituciones Anfitrionas.

\section{Trabajo Final}

El Trabajo Final consistirá en la planificación de un proyecto de internacionalización, para el desarrollo institucional, en base a un problema y/o necesidad detectado a partir de una visita/observación participativa (in situ) a una institución educativa. En caso que no se cuente con la posibilidad de concurrir a una institución se trabajarán estudios de caso brindados por la dirección del programa.

\section{Tipo de Certificación}

Modular y del Programa Completo. Los certificados modulares deberán ser emitidos y firmados en todos los casos por la FFyL- UNCuyo y por la Institución Anfitriona. En el caso 
Programa de formación de posgrado sobre gestión de la internacionalización de la educación superior GIES

UNIVERSIDAD NACIONAL DE CUYO (UN-CUYO, MENDOZA, ARGENTINA) FACULTAD DE FILOSOFÍA Y LETRAS

de la certificación del Programa Completo será emitido por la FFyL - UNCuyo y refrendado por las Instituciones Anfitrionas.

\section{Propuesta Educativa}

Los cursos tendrán un porcentaje presencial (puede ser por streaming) en la sede de los estudiantes, visitas institucionales de una semana en la sede que elija el estudiante (una de las sedes del programa) en el caso del módulo de planificación estratégica, y horas de tutorías virtuales en la plataforma de la Facultad de Filosofía y Letras de la UNCuyo con tutores virtuales disciplinares y no disciplinares.

El porcentaje de cada una de las modalidades de enseñanza y aprendizaje variará según el formato curricular.

La evaluación es por curso. El correspondiente al de Planificación Estratégica de la Internacionalización será un proyecto.

Cada sede podrá incorporar, según la trayectoria en la temática, un docente local.

\section{Ejes/ módulos y temas}

\begin{tabular}{|c|c|c|c|}
\hline Módulo & Descripción general & $\begin{array}{l}\text { Carga } \\
\text { horaria }\end{array}$ & $\begin{array}{l}\text { Docentes } \\
\text { propuestos }\end{array}$ \\
\hline $\begin{array}{l}\text { La internacionalización como } \\
\text { objeto de estudio. Debates y } \\
\text { tendencias }\end{array}$ & $\begin{array}{l}\text { Los procesos de internacionalización e integra- } \\
\text { ción regional: Cooperación Solidaria. Coopera- } \\
\text { ción Sur-Sur. Bloques Regionales. Supranaciona- } \\
\text { les. Tendencias. Políticas de flujo de movilidad de } \\
\text { estudiantes y profesores universitarios de la re- } \\
\text { gión. Geo-política y cooperaciones Sur-Sur o } \\
\text { triangulares: principios y estrategias. Normativa } \\
\text { Internacional, MERCOSUR y de cada país. Los } \\
\text { efectos de convergencia/divergencia que conlleva } \\
\text { la globalización sobre las políticas nacionales y } \\
\text { regionales. Ideas y tendencias hegemónicas y al- } \\
\text { ternativas. Organismos internacionales. La inter- } \\
\text { nacionalización de la educación superior: intensi- } \\
\text { ficar la generación de conocimientos y las capaci- } \\
\text { dades de expertise: observatorios y redes especia- } \\
\text { lizadas: concurrencia y complementariedades. La } \\
\text { internacionalización de las universidades como }\end{array}$ & 15 & \\
\hline
\end{tabular}




\begin{tabular}{|c|c|c|}
\hline & $\begin{array}{l}\text { un proceso dual: dimensión que se expresa hacia } \\
\text { el interior y otra que se expresa hacia el exterior } \\
\text { de las universidades Integración latinoamericana } \\
\text { versus colaboraciones asimétricas. Fuga de cere- } \\
\text { bros. Programas de arraigo/retorno. Condiciones } \\
\text { para la internacionalización Universidades entre } \\
\text { pares y/o colaborativas. } \\
\text { Ciudadanía global. Interacciones entre estudian- } \\
\text { tes y docentes. Los inmigrantes/becarios/estu- } \\
\text { diantes. Competencias globales/internacionales: } \\
\text { relacionamientos / cosmovisiones, comunicación } \\
\text { intercultural; educación emocional /empatía. } \\
\text { Prácticas locales. Articulación con lo local: tu- } \\
\text { rismo académico. ¿Quiénes hacen movilidad? } \\
\text { ¿Qué necesitan? }\end{array}$ & \\
\hline $\begin{array}{l}\text { Políticas y Estrategias de vin- } \\
\text { culación interinstitucional } \\
\text { para la Internacionalización }\end{array}$ & $\begin{array}{l}\text { Modalidades: Modalidad bilateral, trilateral, Mo- } \\
\text { dalidad multilateral Actores y promotores de la } \\
\text { internacionalización: de la reactividad ante las } \\
\text { políticas a una proactividad focalizada. Procesos } \\
\text { institucionales de apropiación de la internaciona- } \\
\text { lización. Los operadores de la internacionaliza- } \\
\text { ción y de otros interesados. Becas y movilidad en } \\
\text { América Latina. Convenios de asistencia téc- } \\
\text { nica y/o capacitación y convenios de coopera- } \\
\text { ción académica; Convenios de cooperación } \\
\text { científica -tecnológica y los convenios de inves- } \\
\text { tigación y desarrollo (I+D); Conformación de } \\
\text { organizaciones, instituciones y asociaciones in- } \\
\text { ternacionales y configuración de Redes, que pue- } \\
\text { den ser de carácter gubernamental o no guberna- } \\
\text { mental. Modalidad bilateral, entre dos Estados: } \\
\text { Cooperación Norte - Sur; Cooperación Sur. La } \\
\text { movilidad estudiantil evidencias estadísticas y se- } \\
\text { ñales de cambio. Concentración de los flujos sa- } \\
\text { lientes y diversificación marginal de sus destinos } \\
\text { Democratizar las oportunidades de movilidad sa- } \\
\text { liente: una cuestión "olvidada" Movilidades cor- } \\
\text { tas y largas: asimetrías e innovaciones Deslocali- } \\
\text { venta de servicios educativos Docentes universi- } \\
\text { tarios y postdoctores: viejos y nuevos sujetos de } \\
\text { movilidad estudiantil en América Latina. Branch }\end{array}$ & 20 \\
\hline
\end{tabular}


Programa de formación de posgrado sobre gestión de la internacionalización de la educación superior -

\section{GIES}

UNIVERSIDAD NACIONAL DE CUYO (UN-CUYO, MENDOZA, ARGENTINA) FACULTAD DE FILOSOFÍA Y LETRAS

\begin{tabular}{|c|c|c|}
\hline & $\begin{array}{l}\text { Campus e inversiones extranjeras en la educación } \\
\text { superior versus sucursales en el extranjero. Pro- } \\
\text { gramas de la UE (programa Erasmus u otros) y } \\
\text { otros bloques y países. Titulaciones conjuntas y } \\
\text { múltiples transnacionales. }\end{array}$ & \\
\hline $\begin{array}{l}\text { Gestión de la información } \\
\text { para la Internacionalización }\end{array}$ & $\begin{array}{l}\text { Mecanismos para la recolección de la informa- } \\
\text { ción Selección de información relevante. Elabo- } \\
\text { ración e implementación políticas instituciona- } \\
\text { les. Información y toma de decisiones. La infor- } \\
\text { mación estratégica para la integración a nivel ins- } \\
\text { titucional. Eficacia en su tratamiento, análisis y } \\
\text { distribución; racionalidad y eficiencia en el uso y } \\
\text { manejo de la información en el tiempo adecuado. } \\
\text { Respuesta a los problemas que se presentan con } \\
\text { ayuda de la tecnología; la comunicación; el área } \\
\text { del saber de la informática de algunos de los pro- } \\
\text { tagonistas del proceso; la utilización de criterios } \\
\text { para la clasificación de la información, las bases } \\
\text { de datos, el control de la fiabilidad de la informa- } \\
\text { ción y la cultura del uso de la misma. La gestión } \\
\text { de la información y gestión estratégica de la in- } \\
\text { formación. La proyección estratégica institucio- } \\
\text { nal sustentada en fuentes fidedignas de informa- } \\
\text { ción. Sistema de Información Estratégico apli- } \\
\text { cado a la gestión universitaria. Universidad Inte- } \\
\text { ligente. }\end{array}$ & 20 \\
\hline $\begin{array}{l}\text { Educación Comparada para } \\
\text { la toma de decisiones en in- } \\
\text { ternacionalización }\end{array}$ & $\begin{array}{l}\text { Aproximaciones a las configuraciones de los sis- } \\
\text { temas de educación superior. Los estudios com- } \\
\text { parados a nivel regional, nacional, internacional y } \\
\text { supranacional. Reseña histórica de la educación } \\
\text { comparada. Definición de la Educación Compa- } \\
\text { rada como una herramienta que permite la des- } \\
\text { cripción, interpretación, yuxtaposición y juicios } \\
\text { de valor sobre los diferentes aspectos de la educa- } \\
\text { ción y de los procesos de internacionalización. } \\
\text { Diseñar y desarrollar investigaciones de educa- } \\
\text { ción comparada, como fuentes para la toma de } \\
\text { decisiones. }\end{array}$ & 20 \\
\hline $\begin{array}{l}\text { Gestión de la Internacionali- } \\
\text { zación del Currículum }\end{array}$ & $\begin{array}{l}\text { Las estrategias de nacionalización, regionaliza- } \\
\text { ción e internacionalización curricular. Adecua- } \\
\text { ciones curriculares. Titulaciones Dobles y Tri- }\end{array}$ & 40 \\
\hline
\end{tabular}




\begin{tabular}{|c|c|c|}
\hline & $\begin{array}{l}\text { ples. Estrategias y normativas para la homologa- } \\
\text { ción y reconocimiento de programas y asignatu- } \\
\text { ras realizados en distintas instituciones a la de } \\
\text { origen. Problemas y obstáculos. Ventajas. Progra- } \\
\text { mas de movilidad. El origen del enfoque de las } \\
\text { competencias. El resurgimiento en el marco de } \\
\text { los procesos de transformación de la Educación } \\
\text { Superior: Espacio Europeo de Educación Supe- } \\
\text { rior (EES). Proyecto Tuning Europa y América } \\
\text { Latina, Comisión SCANS (USA), Proyectos para } \\
\text { la educación media: OCDE, UNESCO, OIT. Ca- } \\
\text { sos Regionales y Nacionales. Documentos nacio- } \\
\text { nales relacionados con las competencias. Contex- } \\
\text { tos de aplicación: académico, laboral-productivo- } \\
\text { empresarial. Términos relacionados: aptitud-ca- } \\
\text { pacidad-habilidad-destreza-talento-objetivos. La } \\
\text { formación de competencias. Planificación, Ense- } \\
\text { ñanza y Evaluación según el enfoque por compe- } \\
\text { tencias. }\end{array}$ & \\
\hline $\begin{array}{l}\text { Gestión Internacionalización } \\
\text { de la Investigación y de la Ex- } \\
\text { tensión }\end{array}$ & $\begin{array}{l}\text { Circulación internacional del conocimiento cien- } \\
\text { tífico: estrategias de publicación. Las revistas in- } \\
\text { dexadas a nivel internacional: un mismo rasero } \\
\text { para disciplinas heterogéneas Las revistas con } \\
\text { factor de impacto en ALC: una solución para la } \\
\text { difusión de los conocimientos producidos local- } \\
\text { mente. Fuga de cerebros, académicos diaspóricos } \\
\text { y migración científica entrante en ALC: Evitar la } \\
\text { fuga de cerebros y migración de retorno. Estre- } \\
\text { char las interacciones con la diáspora. Las moda- } \\
\text { lidades de cooperación internacional universita- } \\
\text { ria y su correspondencia con las funciones uni- } \\
\text { versitarias de docencia, investigación y extensión. }\end{array}$ & 20 \\
\hline $\begin{array}{l}\text { Políticas Lingüísticas en con- } \\
\text { textos de internacionaliza- } \\
\text { ción }\end{array}$ & $\begin{array}{l}\text { La política lingüística de calidad. El plurilin- } \\
\text { güismo se presenta como un eje estratégico en el } \\
\text { proceso de internacionalización de las universi- } \\
\text { dades. Modelo de educación bilingüe/plurilingüe } \\
\text { El inglés como "lingua franca" en el ámbito cien- } \\
\text { tífico-técnico. Plurilingüismo estratégico. Pro- } \\
\text { gramas de instrucción a través del inglés (English } \\
\text { Medium Instruction o EMI) o programas de inte- } \\
\text { gración de contenido y lengua en la educación su- } \\
\text { perior (Language Integrated Learning in Higher }\end{array}$ & 20 \\
\hline
\end{tabular}


Programa de formación de posgrado sobre gestión de la internacionalización de la educación superior GIES

UNIVERSIDAD NACIONAL DE CUYO (UN-CUYO, MENDOZA, ARGENTINA) FACULTAD DE FILOSOFÍA Y LETRAS

\begin{tabular}{|c|c|c|}
\hline & $\begin{array}{l}\text { Education, ICLHE). La enseñanza de los idiomas: } \\
\text { inglés versus multilingüismo. Otras lenguas loca- } \\
\text { les para la comunicación y difusión. Las lenguas } \\
\text { para el trabajo y para la académica. Puesta en fun- } \\
\text { cionamiento de titulaciones bilingües/plurilin- } \\
\text { gües. Plurilingüismo en el contexto de ALC o el } \\
\text { diseño de titulaciones impartidas completamente } \\
\text { en lengua extranjera. Soberanía lingüística. }\end{array}$ & \\
\hline $\begin{array}{l}\text { Aseguramiento transnacional } \\
\text { de la Calidad: Evaluación y } \\
\text { Acreditación Regional e In- } \\
\text { ternacional }\end{array}$ & $\begin{array}{l}\text { La evaluación y la acreditación de instituciones, } \\
\text { programas y sistemas de educación superior La } \\
\text { vinculación de los mecanismos de aseguramiento } \\
\text { de la calidad con el financiamiento, el rol político } \\
\text { de los procesos de integración regional y en la } \\
\text { configuración de los dispositivos de evaluación y } \\
\text { acreditación, el proceso de institucionalización o } \\
\text { de creación de organismos intermedios especiali- } \\
\text { zados en la garantía de la calidad, la articulación } \\
\text { público/privado y entre sectores de instituciones } \\
\text { (universitarias y no universitarias). El MERCO- } \\
\text { SUR y el aseguramiento de su calidad y de la for- } \\
\text { mación de los recursos humanos de los países del } \\
\text { bloque: el MEXA y el ARCUSUR. Los procesos de } \\
\text { aseguramiento de la calidad de la educación en la } \\
\text { Unión Europea y el ALCA. Espacio Europeo de } \\
\text { Educación Superior (EES). Proyecto Tuning Eu- } \\
\text { ropa y América Latina, Comisión SCANS (USA), } \\
\text { Proyectos para la educación media: OCDE, } \\
\text { UNESCO, OIT. Casos Regionales y Nacionales. } \\
\text { UEGM. Acreditar y rankear para dar fe de la cali- } \\
\text { dad. Asegurar la calidad para reordenar la coope- } \\
\text { ración internacional Los rankings en América La- } \\
\text { tina. Normativa actual Reconocimiento de títulos } \\
\text { y periodos de estudio. La compatibilidad y com- } \\
\text { parabilidad a nivel internacional de los sistemas } \\
\text { nacionales de educación superior }\end{array}$ & 40 \\
\hline $\begin{array}{l}\text { Planificación Estratégica de } \\
\text { la Internacionalización: ela- } \\
\text { boración del trabajo final }\end{array}$ & $\begin{array}{l}\text { La incorporación de la internacionalización en la } \\
\text { planeación estratégica en las Instituciones de } \\
\text { Educación Superior (IES) o la Planificación estra- } \\
\text { tégica de la internacionalización. Política de in- } \\
\text { ternacionalización institucional. Metodología } \\
\text { para la planeación estratégica de la Internaciona- }\end{array}$ & 40 \\
\hline
\end{tabular}


lización. La consecución eficiente de metas y objetivos. Matriz FODA. Ejes o lineamientos Estratégicos. Evaluación El rol de las Áreas de Relaciones Internacionales o Cooperación (ORI, DRI, OCI) Propiciar los consensos con directores y actores institucionales para la planeación estratégica del proceso de internacionalización. Desarrollo de Plan y acciones. 